\title{
Novel surface acoustic wave (SAW)-driven closed PDMS flow chamber
}

\author{
Lothar Schmid • Achim Wixforth • David A. Weitz • \\ Thomas Franke
}

Received: 25 May 2011 / Accepted: 20 July 2011/Published online: 19 August 2011

(C) Springer-Verlag 2011

\begin{abstract}
In this article, we demonstrate a novel microfluidic flow chamber driven by surface acoustic waves. Our device is a closed loop channel with an integrated acoustic micropump without external fluidic connections that allows for the investigation of small fluid samples in a continuous flow. The fabrication of the channels is particularly simple and uses standard milling and PDMS molding. The micropump consists of gold electrodes deposited on a piezoelectric substrate employing photolithography. We show that the pump generates a pressure-driven Poiseuille flow, investigate the acoustic actuation mechanism, characterize the flow profile for different channel geometries, and evaluate the driving pressure, efficiency and response time of the acoustic micropump. The fast response time of our pump permits the generation of non-stationary flows. To demonstrate the versatility of the device, we have pumped a red blood cell suspension at a physiological rate of 60 beats/min.
\end{abstract}

Keywords Microfluidics - Lab-on-a-chip - Micropump · Surface acoustic waves (SAW) - Acoustic streaming

Electronic supplementary material The online version of this article (doi:10.1007/s10404-011-0867-5) contains supplementary material, which is available to authorized users.

L. Schmid · A. Wixforth · T. Franke $(\bowtie)$

Microfluidics Group, EP1, Universität Augsburg,

Universitätsstr. 1, Augsburg, Germany

e-mail: thomas.franke@physik.uni-augsburg.de

D. A. Weitz - T. Franke

Department of Physics and School of Engineering and Applied

Sciences, Harvard University, 29 Oxford Street, Cambridge,

MA 02138, USA

\section{Introduction}

Pumps are among the key components to actuate as well as control flow in microfluidic systems. There are a multitude of pumps that have been developed, exploiting various physical effects (Squires and Quake 2005; Stone et al. 2004). Among these are pumps using electrophoretic or dielectrophoretic forces, capillarity, magnetic forces or mechanical techniques creating hydrodynamic pressure gradients to drive fluid flow. The most widely used are electrokinetic and mechanical pumps (Laser and Santiago 2004). However, the flow rate of electrokinetic pumps is relatively low and depends on the electrolyte concentration of the fluid, while mechanical pumps such as syringe pumps can create high pressure gradients but need external fluid tubing and suffer from long response times. Multilayered soft lithography systems have fast response times, however, they also need to be connected to external air pressure generators (Unger et al. 2000). In this communication, we present a pumping technique based on the acoustic streaming effect. This method has the advantages of both tubeless fluid control without any external fluid connection and pumping at high flow rates. The acoustic streaming effect (Eckart 1948; Nyborg et al. 1965) has been recently applied to enhance mixing (Sritharan et al. 2006; Frommelt et al. 2008), pumping (Schneider et al. 2008), and agitation (Franke and Wixforth 2008; Guttenberg et al. 2005; Renaudin et al. 2006) in open microfluidic systems which were chemically modified to achieve hydrophilic/hydrophobic wetting contrast and to confine the liquid. In other open device designs, microchannels were cut by lasers directly into the substrate to guide the fluid (Requa et al. 2009). Recently, a microfluidic device exploiting the acoustic streaming effect indirectly has been developed to actuate fluid in an array of channels 
(Masini et al. 2010). The actuation principle is based on the SAW induced counterflow mechanism and the effect of nebulization anisotropy and relies on the droplet dynamics at the air-liquid interface (Girardo et al. 2008). Using this counterflow effect, where SAW propagation and fluid actuation are oppositely directed (Girardo et al. 2008), the respective authors were able to draw fluid from a reservoir into a channel and move the liquid to a position in the array. In closed systems without a fluid-air interface surface, acoustic waves have proven useful for droplet direction (Franke et al. 2009a) and cell sorting (Franke et al. 2010). The actuation principle of acoustic streaming is independent of $\mathrm{pH}$ or electrolyte concentration and uses low voltages which is important when pumping biological samples and to avoid electrochemical effects. Moreover, the method allows the continuous actuation of non-stationary fluid flow with fast response times. This is important for several applications for instance to experimentally mimic the pulsed beat of blood flow through vessels.

\section{Hybrid chip design}

Our hybrid technique combines widely used PDMS soft lithography and full integration of the fluidic pump on a chip.
Soft lithography using the elastomer polydimethysiloxane (PDMS) enables fabrication of simple and low cost complex microfluidic channel structures (Whitesides and McDonald 2002). The device pumps the fluid in a circle in a closed PDMS channel using acoustic streaming excited by an interdigital transducer (IDT) as shown in the schematic of Fig. 1 (left). The IDT consist of two gold electrodes on a piezoelectric substrate, each having a comb-like structure, which interdigitate at fixed finger repeat distance. The ratio of the sound velocity in the substrate to twice the finger distance defines the operating frequency of the IDT. The chip presented here has a finger spacing of $\sim 13 \mu \mathrm{m}$ and works at $142 \mathrm{MHz}$. Its gold electrodes were produced by vapor deposition and standard lithography. The anisotropic piezoelectric substrate is a $Y$-cut of $\mathrm{LiNbO}_{3}$ with the crystal axis rotated around the $X$-axis by $128^{\circ}$ (128 $Y$-Cut). The fingers of the IDT are carefully aligned perpendicular to the $X$-axis and the alternating RF frequency therefore excites a Rayleigh wave propagating in the direction of the $X$-axis. A fast alternating electric RF-field generates an oscillating displacement with an amplitude in the nm-range which propagates at the velocity of sound on the surface of the piezoelectric substrate. To apply the high frequency voltage, we use a GHz-signal generator (Rohde Schwarz, SML01) and subsequently amplify the signal. To complement the
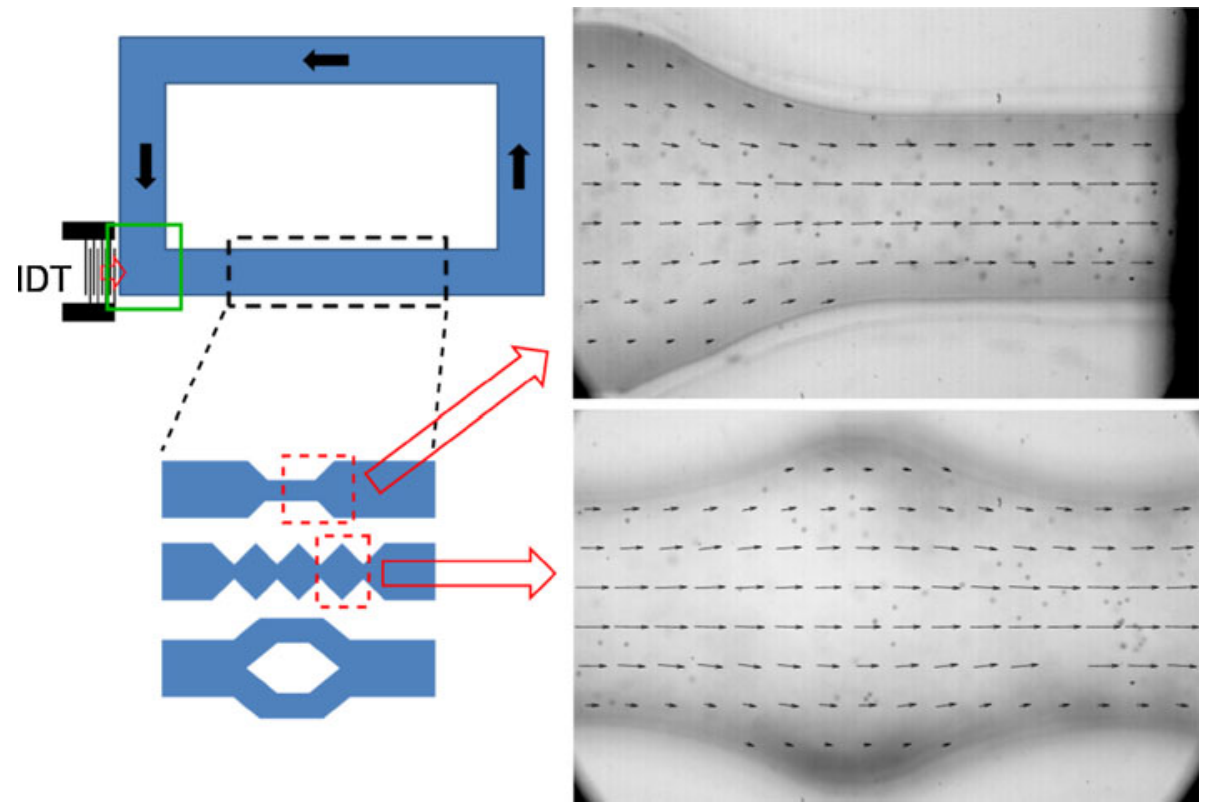

Fig. 1 (Color online) (left) Schematic of the hybrid PDMS-SAW chip as seen from above. The basic channel (width $=1 \mathrm{~mm}$, height $=0.75 \mathrm{~mm}$, loop circumference $=42 \mathrm{~mm}$ ) consists of a closed rectangular PDMS channel and is bonded onto a glass substrate. The IDT is carefully positioned below the channel and pumps the fluid all around the closed channel without inlets or outlets (black arrows indicate flow direction). The IDT consists of 42 fingers, and has an aperture of $624 \mu \mathrm{m}$ and a length of $1233 \mu \mathrm{m}$. It is positioned in parallel to the fluid channel exactly at the PDMS-fluid boundary. Enclosed by a green line is the coupling region of the acoustics to the fluid. A closeup of the coupling is shown in Fig. 2. We have used different channel designs as indicated by the channel sections in the schematic below: a narrowing nozzle-like channel, an oscillating zig-zag channel, and a bifurcated channel. (right) Micrographs of the different channels are superposed with the experimentally measured flow velocity vectors (black arrows) as obtained from particle tracking with focus in the symmetry plane, i.e., middle of the channel (see Supplementary Material for the micrograph of the bifurcated channel) 
device, a microfluidic PDMS channel is assembled and sealed onto a cover slip through covalent bonding after ozone plasma treatment (Whitesides and McDonald 2002; Duffy et al. 1998) and carefully placed on top of the piezoelectric substrate using water as a contact liquid.

When a microfluidic channel is placed on top of the substrate, the Rayleigh wave generates an acoustic wave which couples through the intermediate water layer and the glass bottom of the channel into the fluid, transferring momentum along the direction of propagation and ultimately inducing fluid streaming, as illustrated in Fig. 2 (top). In the actuation region, the SAW creates several flow vortices that we have observed using particle image velocimetry as shown in Fig. 2 (bottom). This complex flow pattern creates a pressure difference that drives the flow along the channel as shown in Fig 1. The flow velocity can be controlled electronically by the SAW power and works without any time lag. Therefore, a time dependent hydrodynamic flow field can be either created by spatially varying the geometry of the channel or alternatively by modulation of the SAW amplitude.

The basic PDMS stamped channel is designed in a rectangular closed loop. To demonstrate the device and its usefulness for biologically relevant geometries, we have replaced a part of the channel by specially designed sections of varying shape. We have used channels with bifurcations and constrictions to mimic the branched vascular system and stenosis.

\section{Flow characterization}

For observation, the assembled PDMS-SAW hybrid device is mounted on the stage of an inverted microscope and imaged by a fast camera (Photron, Fastcam 1024 PCI). We visualize the fluid flow field by adding small latex beads of $\sim 1-\mu \mathrm{m}$ diameter as tracer particles to the microchannel. These beads are fluorescently labeled and were observed and tracked either in phase contrast or by epi-fluorescence as shown in Fig. 1.

To physically characterize the SAW driven flow, we have compared the experimental flow profile of the simplest geometry of a straight rectangular channel with the analytical solution of a pressure driven Stokes flow. With $w, h$ being width and height of the channel the velocity $u$ depends on the pressure gradient $G$ and viscosity $\mu$ (Tabeling 2005):

$$
\begin{aligned}
u(y, z)= & \frac{4 G}{\mu w} \frac{(-1)^{n+1}}{\beta_{n}^{3}} \sum_{n=1}^{\infty}\left(1-\frac{\operatorname{ch} \beta_{n} z}{\operatorname{ch} \beta_{n} \frac{h}{2}}\right) \cos \beta_{n} y, \\
& \text { where } \beta_{n}=(2 n-1) \frac{\pi}{w}
\end{aligned}
$$

Both measured and calculated flow profiles are in excellent agreement as shown in Fig. 3 indicating that the SAW driven

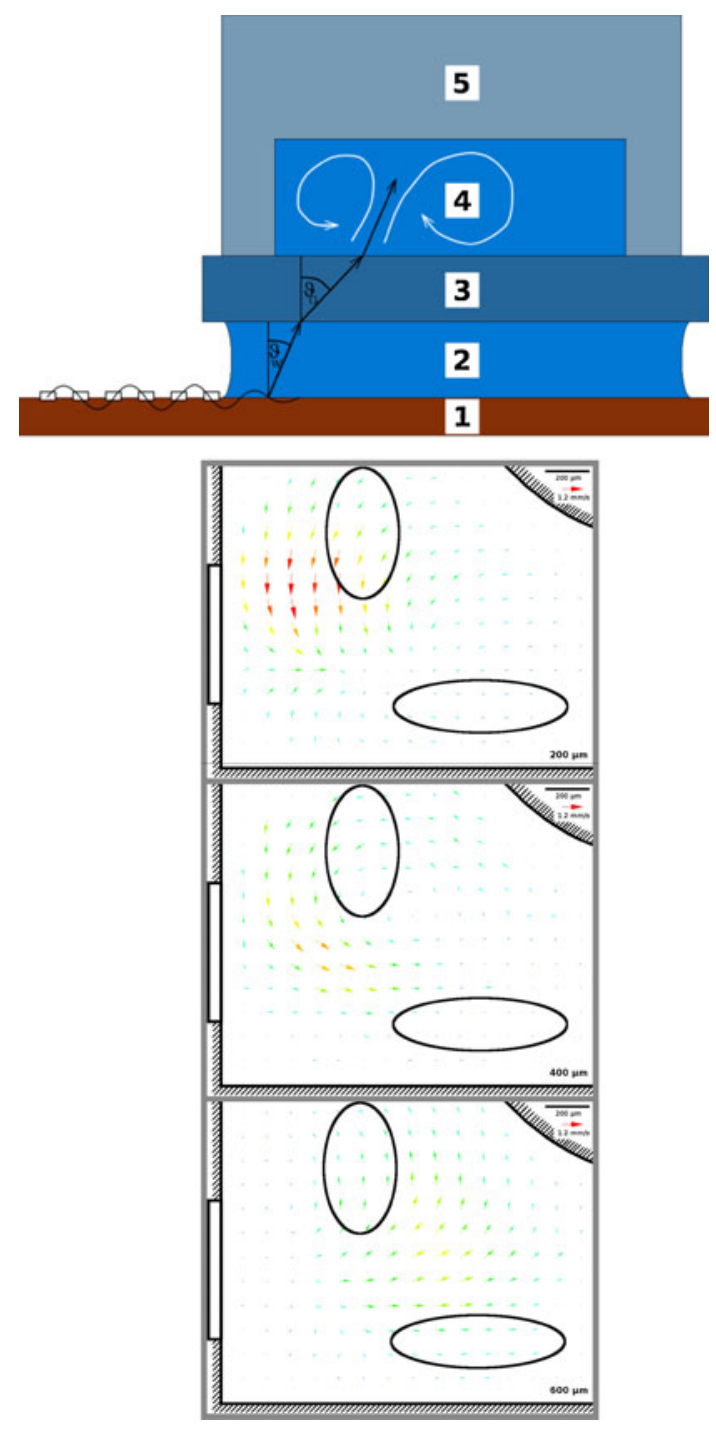

Fig. 2 (Color online) (top) Side view sketch of the path of the acoustic wave coupling into the channel: A surface acoustic wave is excited by the IDT and travels along the lithium niobate substrate (1). It is refracted as a longitudinal wave into the water (2) at a Rayleigh angle $\theta_{\mathrm{W}}=21.8^{\circ}$, while the surface wave on the substrate is attenuated on a characteristic length scale of $325 \mu \mathrm{m}$. The longitudinal acoustic wave passes through the $150-\mu \mathrm{m}$ thick water layer and is subsequently refracted as a transversal wave into the glass coverslide at a Rayleigh angle of angle $\theta_{\mathrm{G}}=54.4^{\circ}$. At the top of the glass slide the wave is refracted again, enters the water-filled channel (4) and transfers momentum to the liquid, causing acoustic streaming as indicated, before it couples into the PDMS layer on top where it is further dampened. (bottom) Flow profile at different $z$ planes at the SAW actuation region (indicated in Fig. 1 (left) as a green square). The black rectangle on the left indicates the position of the IDT. The horizontal vortex (in the $z$ plane) generated by the acoustic wave is clearly visible, and in addition, two vertical vortices are present and denoted by black ellipses. They can be inferred when comparing the flow velocities on the top and bottom planes. The actuation power was $12 \mathrm{dBm}$

flow behaves as a pressure driven flow. Deviations from this velocity profile occur only very close to the IDT where vortices (Franke et al. 2009b) and other complex flow effects 


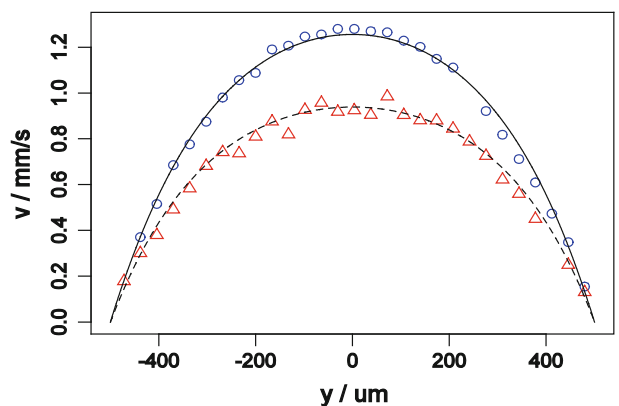

Fig. 3 (Color online) (left) Flow profile measured in the PDMS channel (basic rectangular channel as shown in Fig. 1; width $=1000 \mu \mathrm{m}$, height $=750 \mu \mathrm{m}$ ). The fluid moves in the $x$ direction, $y$ is the horizontal, and $z$ is the vertical direction. The origin of the coordinate system is at the center of the channel. Blue circles represent the flow velocity measured near the channel center $(z=20 \mu \mathrm{m})$, red triangles show the velocity measured at $z=170 \mu \mathrm{m}$. The black line is a fit of the analytical model to the measurement at $z=20 \mu \mathrm{m}$ (blue circles) and yields a pressure

(Frommelt et al. 2008) take over. However, the flow is laminar all over the microchannel because the Reynolds number always obeys $R e<60$ and turbulent flow only occurs when Reynolds numbers are an order of magnitude higher. We determine the pumping efficiency of the device by measuring the pressure dependence on applied electric power of the IDT electrodes. The pressure was determined from our experimental flow profiles by fitting Eq. 1 with $G$ as free parameter. As shown in Fig. 3, this pressure gradient linearly depends on to the electric power for a wide range of power values. Hence, we have a continuous control of the flow velocity from no flow to $4.9 \mathrm{~mm} / \mathrm{s}$ at $29 \mathrm{dBm}$ electrical power, which is comparably high, with a corresponding maximum effective pressure $p=4.8 \mathrm{~Pa}$. The maximum volume flow rate $Q$ at $4.8 \mathrm{~Pa}$ can be calculated from Eq. 1 and yields $Q \sim 0.15 \mathrm{ml} / \mathrm{min}$ (Tabeling 2005) which is in the upper range of dynamic micropumps (Laser and Santiago 2004). As expected for viscosity dominated systems, the efficiency ratio is comparatively low $Q \cdot p / P \sim 10^{-8}$. At higher power, we reach the operating limit of the chip caused by enhanced vibrations.

\section{Acoustic wave coupling}

The power of the acoustic wave in the channel can be determined by calculating the transmission coefficients of the electromechanical transducer and the acoustic wave along its path.

Owing to a mismatch of the IDT impedance, $6.2 \%$ of the electrical energy is reflected at the IDT, leading to a transmission coefficient of $-0.28 \mathrm{~dB}$. One left- and one righttraveling SAW is excited on the substrate, leading to an additional reduction of the actuating power by $-3.01 \mathrm{~dB}$,

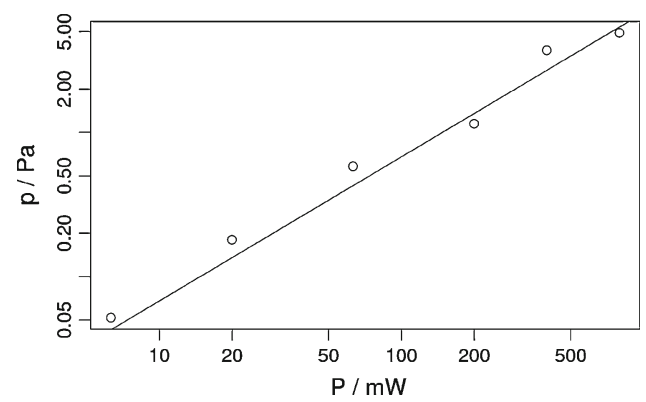

gradient $G=28.5 \mathrm{~Pa} / \mathrm{m}$ as free fitting parameter. The dashed line shows the prediction of the analytical model for off-center flow ( $z=170 \mu \mathrm{m}$ ) using $G$ as determined above. This is in good agreement with measurements (red triangles). (right) Double logarithmic plot of the SAW micropump generated pressure $p$ as calculated from the flow profile at the channel center against the electric SAW power $P$. The pressure is proportional to the electric power (ratio $p / P=(6.78 \pm 0.57) \mathrm{mPa} / \mathrm{mW}$ ). At $29 \mathrm{dBm}$ power, the SAW generates a pressure of approx. $4.8 \mathrm{~Pa}$

since only the latter is transmitted into the water. The SAW is then completely refracted into the liquid with an attenuation length of the surface wave of $l_{\mathrm{SAW}}=325 \mu \mathrm{m}$, which is given by (Dransfeld and Salzmann 1970):

$\alpha=\frac{1}{l_{\mathrm{SAW}}}=\rho_{\mathrm{F}} v_{\mathrm{F}} / \rho_{\mathrm{SAW}} v_{\mathrm{SAW}} \lambda_{\mathrm{SAW}}$

with liquid (subscript F) and substrate (subscript SAW) densities $\rho$, wave velocities $v$, and wave length $\lambda$. The Rayleigh angle $\theta_{\mathrm{W}}=22.1^{\circ}$ can be calculated by Snell's law from the velocity of the SAW $\left(v_{\mathrm{SAW}}=3989 \mathrm{~m} / \mathrm{s}\right)$ (Slobodnik et al. 1973) and the sound velocity in water $\left(v_{\mathrm{F}}=1498 \mathrm{~m} / \mathrm{s}\right)$ (Rossing 2007).

Hence, when passing through the thin water layer, the wave is attenuated by $-0.53 \mathrm{~dB}$.

At the water-glass interface, the wave is refracted once again. In general, when a longitudinal wave is refracted from a liquid into a solid, both a longitudinal and a transversal wave are excited. However, since the longitudinal sound velocity in glass $(5640 \mathrm{~m} / \mathrm{s})$ (Rossing 2007) is much larger than in water, the angle of incidence is larger than the critical angle $\theta_{\text {crit }}=15.4^{\circ}$ of total reflection for longitudinal waves. Therefore, only a transversal wave $(c=3280 \mathrm{~m} / \mathrm{s})$ is excited at a Rayleigh angle of $\theta_{\mathrm{G}}=55.4^{\circ}$. The amplitude ratio of the incident longitudinal wave $\phi$ and transmitted transversal wave $\psi_{1}^{\prime}$ can be calculated from (Brekhovskikh 1980):

$\frac{\psi_{1}^{\prime}}{\phi}=\frac{-2 \rho_{\mathrm{W}} / \rho_{\mathrm{G}} \cdot Z_{\mathrm{t}} \sin ^{2} 2 \theta_{\mathrm{G}}}{Z_{\mathrm{l}} \cos ^{2} 2 \theta_{\mathrm{W}}+Z_{\mathrm{t}} \sin ^{2} 2 \theta_{\mathrm{W}}+Z}$

with impedances $Z$ for the longitudinal sound wave in water, the longitudinal $\left(Z_{1}\right)$ and transversal $\left(Z_{t}\right)$ wave in glass and the corresponding densities $\rho$ and Rayleigh angles $\theta$. 
Exploiting the energy flux density (Landau and Lifshitz 1987):

$q=\frac{1}{2} c \rho_{0} A^{2} n$

with sound velocity $c$, medium density $\rho_{0}$ and wave amplitude $A$ and direction of propagation $n$, the corresponding power transmission coefficient can be calculated to be $T=0.43$, which corresponds to $-3.64 \mathrm{~dB}$. At the upper glass-water interface, the process is reversed, and a longitudinal sound wave is generated with the same transmission coefficient.

Therefore, taking into account the dissipation and reflection in the different media, the total coupling attenuation of the power can be estimated to be $\Delta D=-11.1 \mathrm{~dB}$.

\section{The origin of streaming}

The theoretical background of acoustic streaming has been thoroughly investigated by Nyborg et al. (1965): a sound wave traveling through a liquid is damped due to relaxational dissipation. Dissipation causes a volume force on the liquid that is proportional to the frequency-dependent damping coefficient and acts in the direction of wave propagation. Considering a plane wave traveling in $x$-direction with characteristic attenuation length $l_{\mathrm{a}}$ and Amplitude A:

$u_{1}=A \mathrm{e}^{-x / l_{\mathrm{a}}} \cos (\omega \mathrm{t}-k x)$

This force is given by:

$F_{x}=\rho_{0} A^{2} \mathrm{e}^{-2 x / l_{\mathrm{a}}} / l_{\mathrm{a}}$

The attenuation length at $142 \mathrm{MHz}$ in water is $l_{\mathrm{a}}=1.24 \mathrm{~mm}$ (Coates 1989; Hagen et al. 2004). The amplitude of the wave can be calculated from the wave power $P$ and area $S$ :

$P=q \cdot S=\frac{1}{2} c \rho_{0} A^{2} n \cdot S$

which yields a volume force

$F_{x}=\frac{2 P}{c S l_{\mathrm{a}} \cos \theta} \mathrm{e}^{-2 x / l_{\mathrm{a}}}$

Equation 8 gives a linear relation between acoustic power and bulk force acting on the fluid. Because both the relation of electric and acoustic power and the Stokes equation governing the fluid flow are linear, we expect a linear relation between electric power and pressure. This linearity has been observed in our experiments as shown in Fig. 3 (right).

To assess the pressure that drives the fluid, we employ a maximum electrical power of $29 \mathrm{dBm}$ corresponding to an acoustic power $P$ of $17.9 \mathrm{dBm}$; we estimate the cross section area $S$ from the IDT aperture $b=624 \mu \mathrm{m}$ and the SAW attenuation length as $S=b \cdot l_{\mathrm{SAW}}$ cited above. The volume force at the bottom of the channel then is $F_{x}=176 \mathrm{kN} / \mathrm{m}$ (Laser and Santiago 2004) at an amplitude $A=0.66 \mathrm{~m} / \mathrm{s}$. By integrating along the direction of propagation from the bottom of the channel $(h=0)$ to the top $(h=750 \mu \mathrm{m})$, the resulting pressure difference $\Delta p$ can be calculated:

$\Delta p=\int_{0}^{h / \cos \theta_{\mathrm{w}}} \mathrm{dx} F_{x}(x) \approx 160 \mathrm{~Pa}$

This pressure difference drives the vortices in the pumping region and causes an effective pumping pressure of $4.8 \mathrm{~Pa}$. The significant difference between these two values is in good agreement with the observed flow velocities in the vortex, which are one order of magnitude higher than in the channel.

The driving pressure difference is a direct consequence of the dissipation in the channel that depends on the frequency. Because dissipation occurs in the contact liquid as well and does not contribute to the driving pressure in the channel there is an optimal frequency for a given geometry (ratio of channel to contact liquid height). For the device we present here the optimal attenuation length is $0.7 \mathrm{~mm}$ and $90 \%$ of that value is still obtained in the range of $0.4-1.2 \mathrm{~mm}$. This corresponds to a frequency range of 130-240 MHz (Coates 1989).

\section{Time-dependent flow}

Another important feature of a pump is its ability to create time dependent flow. Many commonly used pumps have surprisingly long transients. For example, syringe pumps have response times that can range from several tenths of seconds to even minutes. Response times of the SAW driven device are determined by application of a square wave modulated HF signal to the electrodes and the corresponding flow relaxation is measured for different channel dimensions $(w=500 \mu \mathrm{m}$ and $w=1000 \mu \mathrm{m}$ at $h=750 \mu \mathrm{m}$ ) as shown in Fig. 4. Because comparison with a pressure driven flow is useful, as discussed above, we compare the experimental results with a theoretical estimation of the relaxation of a non-stationary flow in response to a pressure jump. For simplification, we consider a resting fluid in a cylinder of radius $a$ and turn on the pressure gradient $G$ at time $t=0$. With no-slip boundary conditions the velocity yields (Batchelor et al. 2000), 


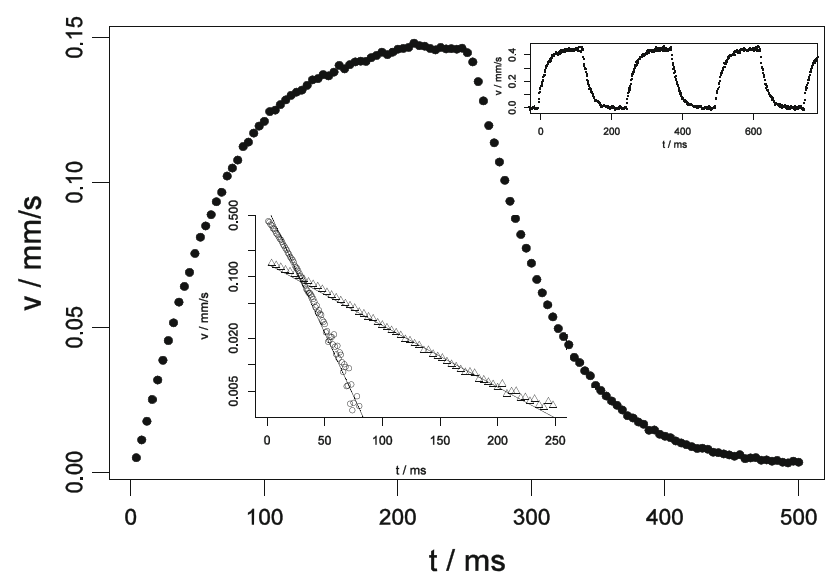

Fig. 4 Response of the flow velocity to a $2 \mathrm{~Hz}$ square-wave modulation of the SAW $(20.5 \mathrm{~mW}, 13.1 \mathrm{dBm})$. The top right inset shows the time-dependent flow velocity of a $4 \mathrm{~Hz}$ modulation in a smaller channel $(63.5 \mathrm{~mW}, 18.0 \mathrm{dBm})$. After voltage cut-off (between 250 and $500 \mathrm{~ms}$ ), the flow velocity decays exponentially. The inset below shows on a logarithmic scale the decay in two different channels both of height $=0.75 \mathrm{~mm}$ and a width of 1 and $0.5 \mathrm{~mm}$, respectively. The exponential fits results in a decay time $\tau=61.7 \pm 0.4 \mathrm{~ms}$ for the wider channel and a decay time $\tau=15.1 \pm 0.4 \mathrm{~ms}$ for the smaller channel. A theoretical analysis yields decay times of 58 and $25 \mathrm{~ms}$ (see text)

$u(r, t)=\frac{G}{4 \mu}\left(a^{2}-r^{2}\right)-\frac{2 G a^{2}}{\mu} \sum_{n=1}^{\infty} \frac{J_{0}\left(\lambda_{n} \frac{r}{a}\right)}{\lambda_{n}^{3} J_{1}\left(\lambda_{n}\right)} \exp \left(-\lambda_{n}^{2} \frac{v t}{a^{2}}\right)$

$J_{n}$ being the Bessel functions and $\lambda_{n}$ the roots of $J_{O}$ $(\lambda)=0$. The first term $n=1$ of the series is the slowest decaying component and can be used to estimate the response time. We approximate the rectangular channel of the experiment by the cylinder symmetric solution of Eq. 10. Using $a=\frac{1}{4} \sqrt{b h}$, we calculate $\tau=58 \mathrm{~ms}$ and $\tau=25 \mathrm{~ms}$ for the wide and narrow channels, respectively, in good agreement with the experimental values of 62 and $15 \mathrm{~ms}$.

\section{Pumping biofluids}

Finally, we demonstrated that the pumping device presented here is able to pump biologically relevant samples such as red blood cell solution in $300 \mathrm{mOsm}$ phosphate buffered saline [Dulbecco's PBS $(1 \times)$, PAA Laboratories $\mathrm{GmbH}, 0.2 \mathrm{~g} / \mathrm{l} \mathrm{KCl}, 0.2 \mathrm{~g} / 1 \mathrm{~K}_{3} \mathrm{PO}_{4}, 8.0 \mathrm{~g} / \mathrm{l} \mathrm{NaCl}, 1.15 \mathrm{~g} / \mathrm{l}$ $\left.\mathrm{Na}_{2} \mathrm{HPO}_{4}\right]$. We exploit the fast response time of our device to simulate blood flow at a frequency of 60 beats/min by amplitude modulation of the voltage with a square wave signal of $1 \mathrm{~Hz}$ using a frequency generator (Rohde Schwarz, SML01) and to pump in the circulatory channel system (see Movie in Supplementary Material).
In conclusion, we have given a detailed description of the working principles of the SAW-driven flow chamber. We have also shown that our SAW hybrid device is a versatile platform to mimic different stationary and nonstationary fluid flow fields. The complete system is very compact with the SAW micropump fully integrated on a chip without any external fluid tubing preventing crosscontamination of the sample. This allows exceptional electronic control of the fluid flow. The circulatory, closed loop design of the device will enable the integration and combination of several micropumps and fluid interconnections to perform more complex processes on a single chip; this could be useful in a variety of applications such as evolutionary biotechnology or lab-on-a-chip systems.

Acknowledgments This study was supported by the Bayerische Forschungsstiftung, the German Excellence Initiative via Nanosystems Initiative Munich (NIM), the Center for Nanoscience (CeNS), and the German Academic Exchange Service DAAD.

\section{References}

Batchelor GK (2000) An introduction to fluid dynamics. Cambridge University Press, Cambridge

Brekhovskikh M (1980) Waves in layered media. Academic Press, New York

Coates RFW (1989) Underwater acoustic waves. Wiley, New York

Dransfeld K, Salzmann E (1970) Excitation, detection, and attenuation of high-frequency elastic surface waves. In: Mason WP, Thurston RN (eds) Physical acoustics. Academic Press, New York, pp 219-272

Duffy DC, McDonald JC, Schueller OJA, Whitesides GM (1998) Rapid prototyping of microfluidic systems in poly (dimethylsiloxane). Anal Chem 70(23):4974-4984

Eckart C (1948) Vortices and streams caused by sound waves. Phys Rev 73(1):68

Franke T, Wixforth A (2008) Microfluidics for miniaturized laboratories on a chip. ChemPhysChem 9(15):2140-2156

Franke T, Abate AR, Weitz DA, Wixforth A (2009a) Surface acoustic wave (SAW) directed droplet flow in microfluidics for PDMS devices. Lab Chip. doi:10.1039/b906819h

Franke T, Braunmüller S, Frommelt T, Wixforth A (2009b) Sorting of solid and soft objects in vortices driven by surface acoustic waves. In: Proceedings of SPIE 2009

Franke T, Braunmuller S, Schmid L, Wixforth A, Weitz DA (2010) Surface acoustic wave actuated cell sorting (SAWACS). Lab Chip 10(6):789-794

Frommelt T, Kostur M, Wenzel-Schafer M, Talkner P, Hanggi P, Wixforth A (2008) Microfluidic mixing via acoustically driven chaotic advection. Phys Rev Lett 100(3):034502-034504

Girardo S, Cecchini M, Beltram F, Cingolani R, Pisignano D (2008) Polydimethylsiloxane- $\mathrm{LiNbO}_{3}$ surface acoustic wave micropump devices for fluid control into microchannels. Lab Chip 8(9):1557-1563

Guttenberg Z, Muller H, Habermuller H, Geisbauer A, Pipper J, Felbel J, Kielpinski M, Scriba J, Wixforth A (2005) Planar chip device for PCR and hybridization with surface acoustic wave pump. Lab Chip 5(3):308-317 
Hagen R, Behrends R, Kaatze U (2004) Acoustical properties of aqueous solutions of urea: reference data for the ultrasonic spectrometry of liquids. J Chem Eng Data 49(4):988-991

Landau LD, Lifshitz EM (1987) Course of theoretical physics. Pergamon Press, New York

Laser DJ, Santiago JG (2004) A review of micropumps. J Micromech Microeng 14:R35-R64

Masini L, Cecchini M, Girardo S, Cingolani R, Pisignano D, Beltram F (2010) Surface-acoustic-wave counterflowmicropumps for onchip liquid motion control in two-dimensional microchannel arrays. Lab Chip 10(15):1997-2000

Nyborg WL (1965) Acoustic streaming. Academic Press, New York

Renaudin A, Tabourier P, Zhang V, Camart JC, Druon C (2006) SAW nanopump for handling droplets in view of biological applications. Sensors Actuators B 113(1):389-397

Requa MV, Fraikin JL, Stanton MA, Cleland AN (2009) Nanoscale radiofrequency impedance sensors with unconditionally stable tuning. J Appl Phys 106:074308

Rossing TD (2007) Springer handbook of acoustics. Springer, New York

Schneider MF, Guttenberg Z, Schneider SW, Sritharan K, Myles VM, Pamukci U, Wixforth A (2008) An acoustically driven microliter flow chamber on a chip (muFCC) for cell-cell and cell-surface interaction studies. ChemPhysChem 9(4):641-645

Slobodnik AJ, Delmonico RT, Conway ED (1973) Microwave acoustics handbook, vol 2. In: Physical Sciences Research Papers Air Force Cambridge Research Labs, Hanscom AFB, MA

Squires TM, Quake SR (2005) Microfluidics: fluid physics at the nanoliter scale. Rev Modern Phys 77(3):977-1026

Sritharan K, Strobl CJ, Schneider MF, Wixforth A, Guttenberg Z (2006) Acoustic mixing at low Reynold's numbers. Appl Phys Lett 88(5):054102-054103

Stone HA, Stroock AD, Ajdari A (2004) Engineering flows in small devices microfluidics toward a lab-on-a-chip. Annu Rev Fluid Mech 36:381-411

Tabeling P (2005) Introduction to microfluidics. Oxford University Press, Oxford

Unger MA, Chou HP, Thorsen T, Scherer A, Quake SR (2000) Monolithic microfabricated valves and pumps by multilayer soft lithography. Science 288(5463):113

Whitesides GM, McDonald C (2002) Poly(dimethylsiloxane) as material for fabricating microfluidic devices. Acc Chem Res 35(7):491-499 